\title{
MECANISMOS DE VIRULÊNCIA DO SARS-COV-2 E AS COMPLICAÇÕES NO PROGNÓSTICO DE PACIENTES COM DIABETES MELLITUS: UMA REVISÃO DE LITERATURA
}

\section{SARS-COV-2 VIRULENCE MECHANISMS AND COMPLICATIONS IN THE PROGNOSIS OF PATIENTS WITH DIABETES MELLITUS: A LITERATURE REVIEW}

\author{
Fabiana Santana Britto ${ }^{1}$ \\ Mayara Conceição dos Santos ${ }^{2}$ \\ Klleyza Santos Conceição ${ }^{3}$ \\ Tasciano dos Santos Santa Izabel ${ }^{4}$ \\ Danrley Oliveira Carneiro ${ }^{5}$
}

RESUMO: Introdução: As infecções humanas por Coronavírus são conhecidas há décadas, em específico, a Síndrome Respiratória Aguda Grave (SARS) e a Síndrome Respiratória do Oriente Médio (MERS). Entretanto, a partir de 2019, um novo coronavírus denominado de SARS-CoV-2 foi disseminado por vários países, tomando uma proporção sem precedentes. A partir disso, com o aumento no número de infectados e mortos em diferentes continentes, resultantes da epidemia em Março de 2020 a Organização Mundial da Saúde OMS decretou oficialmente que o surto da doença tratava-se de uma pandemia de caráter mundial. Objetivo: Realizar uma revisão de literatura acerca dos mecanismos de virulência do SARS-Cov-2, bem como as suas complicações acerca do prognóstico de pacientes portadores de Diabetes Mellitus. Metodologia: Foram realizadas buscas bibliográficas eletrônicas utilizando descritores previamente selecionados nas bases de dados Pubmed, Lilacs e Scielo que abordassem o tema proposto publicados no período de 2010 a 2021. Resultados: Foram selecionados o8 artigos e os achados dos estudos demonstraram que o SARSCoV-2 gera diversas consequências nos pacientes com DM, uma vez propicia um ambiente inflamatório que resulta em infecções severas e de difícil tratamento. Conclusão: Portanto, pode-se verificar que a inflamação metabólica provocada pelo SARS-CoV-2 resulta em repercussões negativas no prognóstico de paciente portador de DM, dificultando assim, o processo de cura e recuperação do indivíduo, levando-o este a um pior desfecho.

\footnotetext{
${ }^{1}$ Acadêmica do curso de graduação em Biomedicina da Faculdade UNEF de Feira de Santana, Feira de Santana-BA, Brasil.

${ }^{2}$ Acadêmica do curso de graduação em Biomedicina da Faculdade UNEF de Feira de Santana, Feira de Santana-BA, Brasil.

3 Acadêmica do curso de graduação em Biomedicina da Faculdade UNEF de Feira de Santana, Feira de Santana-BA, Brasil.E-mail: klleyza6@hotmail.com.

${ }_{4}^{4}$ Doutor em Botânica, professor do curso de Biomedicina da Faculdade UNEF de Feira de Santana, Feira de Santana-BA, Brasil.

5 Co-Orientador e Biomédico.
} 
Palavras-chaves: Virulência, Coronavírus, Complicações do Diabetes, Prognóstico, Diabetes Mellitus.

ABSTRACT: Introduction: Human Coronavirus infections have been known for decades, specifically, the Severe Acute Respiratory Syndrome (SARS) and the Middle East Respiratory Syndrome (MERS). However, from 2019 onwards, a new coronavirus called SARS-CoV-2 has spread to several countries, taking an unprecedented proportion. From then on, with the huge increase in the number of infected and dead in different continents, resulting from the epidemic, in March 2020 the World Health Organization WHO officially decreed that the outbreak of the disease was a worldwide pandemic. Objective: To carry out a literature review about the virulence mechanisms of SARS-Cov-2, as well as its complications regarding the prognosis of patients with Diabetes Mellitus. Methodology: Electronic bibliographic searches were performed using descriptors previously selected in the Pubmed, Lilacs and Scielo databases that addressed the proposed theme in the period 2010 to 2021. Results: 08 articles were selected and the findings of the studies showed that the SARS-CoV- 2 ends up causing great consequences in patients with DM, as it provides an inflammatory environment that results in severe infections that are difficult to treat. Conclusion: Therefore, it can be seen that the metabolic inflammation caused by SARS-CoV-2 results in negative repercussions on the prognosis of patients with DM, thus hindering the individual's healing and recovery process, leading him to a worst outcome.

Keywords: Virulence, Coronavirus, Diabetes Complications, Prognosis, Diabetes Mellitus.

\section{INTRODUÇÃO}

As infecções em humanos causadas por Coronavírus são conhecidas há algumas décadas, em específico, a Síndrome Respiratória Aguda Grave (SARS) e a Síndrome Respiratória do Oriente Médio (MERS). Entretanto, a partir de 2019, tendo início na China, um novo coronavírus denominado de SARS-CoV-2 passou a ter circulação no mundo e em pouco tempo foi sendo disseminado por várias cidades, e atingindo outros países, tomando uma proporção sem precedentes (ANGHEBEM; REGO; PICHETH, 2020; HIRANO; MURAKAMI, 2020; ANDERSEN et al., 2020).

Com o aumento no número de infectados e mortos resultantes da epidemia em Março de 2020 a Organização Mundial da Saúde OMS decretou oficialmente que o surto da doença tratava-se de uma pandemia de caráter mundial (OMS, 2020).

O SARS-CoV-2, agente causador da COVID-19, é um vírus zoonótico que tem como material genético o RNA, pertencente à família Coronaviridae que pode 
provocar doenças do trato respiratório, intestinais, bem como hepáticas e neurológicas associados às infecções agudas e graves (NOBRE et al., 20I4; BRASIL, 2019; BRASIL, 2020; ZHU et al., 2020).

As infecções respiratórias agudas (IRA) existentes no quadro permeado pela COVID-I9 representam grandes causas de morbidade e mortalidade, e que predispõe os indivíduos a infecções bacterianas secundárias. Dentre os casos fatais ou com maior gravidade, estes acabam prevalecendo em indivíduos com certas comorbidades como: Hipertensão, doenças cardiovasculares, câncer, doenças pulmonares crônicas, além do diabetes, uma vez que, a questão hiperglicêmica revela ser um grande fator de mortalidade e morbidade para tal infecção (YANG et al., 2006; NOBRE et al., 2014; ANGHEBEM; REGO; PICHETH, 2020; GUAN et al., 2020).

A Diabetes Mellitus (DM) caracteriza-se como uma Síndrome de etiologia múltipla, sendo causada por defeitos na secreção e/ou ação da insulina, corroborando assim, em estados de hiperglicemia crônica. O quadro hiperglicêmico favorece as vias metabólicas responsáveis pela formação de produtos finais de glicação avançada, AGEs (Produtos finais de Glicação Avançada), além da liberação de citocinas proinflamatórias e estresse oxidative (BARBOSA; CAMBOIM, 20I6; SBD, 2019; ANGHEBEM; REGO; PICHETH, 2020).

As características inflamatórias evidenciadas na DM e na COVID- i9 acabam por desencadear um desequilíbrio no processo de coagulação e fibrinólise, elevandose assim as concentrações dos fatores de coagulação, além da inibição do sistema de fibrinólise, o que pode gerar um estado pró-trombótico hipercoagulável. Portanto, o SARS-CoV-2 provoca grandes alterações no metabolismo e na homeostasia da glicose, o que reflete maior severidade das complicações aos portadores de diabetes (ANGHEBEM; REGO; PICHETH, 2020).

A hiperglicemia crônica, característica da DM, em associação com outras alterações metabólicas acabam provocando alterações a nível imunológico, proporcionando um ambiente inflamatório que favorece a infecções severas e de tratamento dificultoso, refletindo-se assim, em um longo período de internação. Neste sentido, o presente estudo tem como objetivo realizar uma revisão de literatura 
acerca dos mecanismos de virulência do SARS-Cov-2, bem como as suas complicações acerca do prognóstico de pacientes portadores de Diabetes Mellitus.

\section{MATERIAL E MÉTODOS}

O Presente estudo trata-se de uma Revisão de literatura de natureza qualitativa acerca dos mecanismos de virulência do SARS-CoV-2, bem como as suas complicações no prognóstico de pacientes com Diabetes Mellitus. Foram utilizadas as respectivas bases de dados: Pubmed, Lilacs (Literatura LatinoAmericana e do Caribe em Ciências da Saúde), Scielo (ScientificEletronic Library Online) e Medline (Medical Literature Analysis and Retrieval System Online), para busca de artigos que abordassem o tema proposto no período de 2010 a 2021.

Foram utilizados os descritores de assunto na língua portuguesa: "Virulência", “Coronavírus", "Complicações do Diabetes”, "Prognóstico", "Diabetes Mellitus” e na língua inglesa: "Virulence”, "Coronavirus", "Diabetes Complications”, "Prognosis", “Diabetes Mellitus”, sendo que estes foram previamente testados no DeCS (Descritores em Ciências da Saúde).

Para a escolha dos artigos foram realizadas análises individuais dos resumos para verificar quais estudos eram pertinentes ao tema desta revisão. Desta forma, como critérios de inclusão foram selecionados artigos que abordassem a temática, escritos em português, inglês ou espanhol na íntegra na versão online.

Os principais achados obtidos foram sumarizados no formato de quadro (Quadro I) e posteriormente discutidos.

\section{RESULTADOS E DISCUSSÃO}

Foram encontrados inicialmente 6o artigos e após realização dos critérios de inclusão e exclusão, oito artigos foram incluídos para compor este estudo. Dentre os principais achados identificados nos estudos foi encontrado que o SARS-CoV-2 provoca grandes consequências nos pacientes com DM, uma vez que há uma agressão nas células das ilhotas pancreáticas, o que promove alterações no metabolismo e homeostasia da glicose, além de propiciar um ambiente inflamatório por meio da ação de citocinas. Neste contexto, a associação de tais elementos pode causar infecções 
severas e de difícil tratamento, o que amplia a severidade de complicações, favorecendo assim, um pior prognóstico nesses pacientes.

Quadro I- Artigos que abordaram os mecanismos de virulência do SARS-COV-2, bem como as complicações no prognóstico de pacientes com diabetes mellitus.

\begin{tabular}{|c|c|c|c|c|c|}
\hline $\begin{array}{c}\text { Autor/ } \\
\text { Ano }\end{array}$ & Revista & $\begin{array}{l}\text { Título } \\
\text { do } \\
\text { Artigo }\end{array}$ & Objetivo & Método & Resultados \\
\hline $\begin{array}{l}\text { Rodriguez, } \\
\text { zor } 8 .\end{array}$ & $\begin{array}{l}\text { Repositó } \\
\text { rio } \\
\text { Instituciona1 } \\
\text { UAM. }\end{array}$ & $\begin{array}{l}\text { Papel das } \\
\text { Viroporinas } \\
\text { E, ga e } 8=\text { do } \\
\text { SARS } \\
\text { Coronavírus } \\
\text { na re- } \\
\text { plicação e } \\
\text { viru-lência. }\end{array}$ & $\begin{array}{l}\text { Estudar a relevincia } \\
\text { das viropurinas e, } \\
\text { mais } \\
\text { especificamente, de } \\
\text { seus ICs e FBMs, na } \\
\text { replicação e } \\
\text { virulência do SARS- } \\
\text { CoV combinando } \\
\text { com estratégias } \\
\text { multidisciplinares. }\end{array}$ & $\begin{array}{l}\text { A linhag } \\
\text { em celular High } \\
\text { Pive derivada de } \\
\text { células ovarianas } \\
\text { do looper de } \\
\text { repolho, Vero E6 } \\
\text { derivada de rim } \\
\text { de macaco e } \\
\text { derivadas de } \\
\text { hamster BHK- } \\
\text { ar foram obtidas } \\
\text { partie de a } \\
\text { Laboratório. } \\
\text { Tais células } \\
\text { foram } \\
\text { adase cultiv } \\
\text { Analisadas o } \\
\text { seu DNA, logo } \\
\text { após Forsm } \\
\text { obtidos SARS- } \\
\text { CoVs re } \\
\text { - combi } \\
\text { nantes, com a } \\
\text { respectiva } \\
\text { manipulação } \\
\text { dos RNAs, } \\
\text { purificação e } \\
\text { nálise de } \\
\text { proteinas. }\end{array}$ & 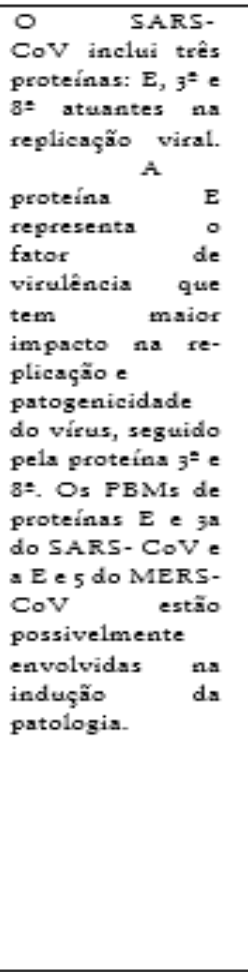 \\
\hline $\begin{array}{l}\text { Aquino } \\
\text { et al., } \\
2020 .\end{array}$ & $\begin{array}{l}\text { Brazilian } \\
\text { Journal } \\
\text { of health } \\
\text { Re view. }\end{array}$ & $\begin{array}{l}\text { Influência } \\
\text { do DMz } \\
\text { e do } \\
\text { controle } \\
\text { glicêmico } \\
\text { no pr } \\
\text { og-nóstico } \\
\text { de } \\
\text { pacientes } \\
\text { infectados } \\
\text { por CO- } \\
\text { VID-rg. }\end{array}$ & $\begin{array}{l}\text { Verificar a } \\
\text { influéncis do } \\
\text { DMz no } \\
\text { prognóstico ds } \\
\text { infeç̧ } \\
\text { ão por do, } \\
\text { COVID- ro } \\
\text { assim como o } \\
\text { impacto } \\
\text { controle gli- } \\
\text { cêmico nos }\end{array}$ & $\begin{array}{l}\text { Revisão de li- } \\
\text { teratura. }\end{array}$ & $\begin{array}{l}\text { A adaptação } \\
\text { metabólica nos } \\
\text { pacientes } \\
\text { diabéticos de- } \\
\text { monstra estar } \\
\text { prejudicada } \\
\text { no contexto } \\
\text { do COVID- } \\
\text { rg, o que } \\
\text { predispõe o } \\
\text { indivíduo à } \\
\text { infeç̧ão, sur- }\end{array}$ \\
\hline & & & $\begin{array}{l}\text { desfechos a- } \\
\text { presentados por } \\
\text { esses pa- } \\
\text { cientes. }\end{array}$ & & $\begin{array}{l}\text { gimento de } \\
\text { complicações, } \\
\text { além de estar } \\
\text { atrelado a um } \\
\text { pior prognó } \\
\text { stico e controle } \\
\text { de glicemis. }\end{array}$ \\
\hline
\end{tabular}

Fonte: Autores. 
Quadro II- Artigos que abordaram os mecanismos de virulência do SARS-COV-2, bem como as complicações no prognóstico de pacientes com diabetes mellitus (continuação).

\begin{tabular}{|c|c|c|c|c|c|}
\hline $\begin{array}{c}\text { Autor/ } \\
\text { Ano }\end{array}$ & Revista & $\begin{array}{r}\text { Título do } \\
\text { Artigo }\end{array}$ & Objetivo & Métodos & Resultados \\
\hline $\begin{array}{l}\text { Anghe } \\
\text { bem; Rego; } \\
\text { Picheth,202 } \\
\text { o. }\end{array}$ & $\begin{array}{l}\text { Rev. } \\
\text { Bras. } \\
\text { Anal. } \\
\text { Clin. }\end{array}$ & $\begin{array}{l}\text { COVID-ı9 } \\
\text { e Diabetes: a } \\
\text { relação en- } \\
\text { tre duas } \\
\text { pandemias } \\
\text { distintas. }\end{array}$ & $\begin{array}{l}\text { Destacar } \\
\text { aspectos } \\
\text { da } \\
\text { relação entre } \\
\text { CO-VID- } \\
\text { I9 e } \\
\text { diabetes. }\end{array}$ & $\begin{array}{l}\text { Revisão de } \\
\text { Literatura. }\end{array}$ & 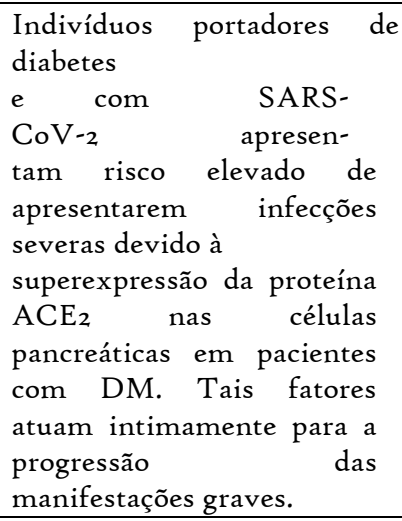 \\
\hline
\end{tabular}

Fonte: Autores.

\begin{tabular}{|c|c|c|c|c|c|}
\hline $\begin{array}{l}\text { Autor/ } \\
\text { Ano }\end{array}$ & Revista & $\begin{array}{c}\text { Título } \\
\text { do Artigo }\end{array}$ & Objetivo & Métodos & Resultados \\
\hline $\begin{array}{l}\text { Brito et al., } \\
2020 .\end{array}$ & $\begin{array}{c}\text { J. Bras. Patol. } \\
\text { Med. } \\
\text { Lab. }\end{array}$ & $\begin{array}{l}\text { Mecanismos } \\
\text { imunopatológicos } \\
\text { emvolvidos na } \\
\text { infecção por } \\
\text { SARS-CoV- } \\
\text { 2. }\end{array}$ & $\begin{array}{l}\text { Reunir e } \\
\text { discutir as } \\
\text { evidências } \\
\text { disponíveis } \\
\text { sobre os } \\
\text { principais } \\
\text { Mecanismo } \\
\text { s de } \\
\text { transmis- } \\
\text { são imu- } \\
\text { nopatológic } \\
\text { os envolvi- } \\
\text { dos na } \\
\text { infec-ção } \\
\text { pelo } \\
\text { SARS- } \\
\text { CoV-2. }\end{array}$ & $\begin{array}{l}\text { Revisão } \\
\text { Narrativa } \\
\text { de } \\
\text { Literatura. }\end{array}$ & $\begin{array}{l}\text { Estudos imuno- patológicos } \\
\text { su- } \\
\text { gerem que a hiper citocine- } \\
\text { mia promove lesão do tecido } \\
\text { pulmonar, } \\
\text { comprometendo } \\
\text { órgãos e siste- } \\
\text { mas. Acerca da } \\
\text { resposta humoral, os } \\
\text { elevados títulos de linfócitos } \\
\text { B e anticorpos podem estar } \\
\text { ligados à grávidade da } \\
\text { doença. }\end{array}$ \\
\hline $\begin{array}{l}\text { Muniyappa; } \\
\text { Gubbi, } \\
2020 .\end{array}$ & $\begin{array}{c}\text { Jornal } \\
\text { Americano } \\
\text { de } \\
\text { Psicologia } \\
\text { Endocrinol } \\
\text { ogia e } \\
\text { Metabolismo. }\end{array}$ & $\begin{array}{l}\text { Pandemia de } \\
\text { COVID- } \\
\text { I9, corona- vírus } \\
\text { e dia- betes mel- } \\
\text { litus. }\end{array}$ & $\begin{array}{l}\text { Discutir os } \\
\text { mecanismos } \\
\text { potenciais } \\
\text { pelos quais o } \\
\text { diabetes } \\
\text { modula as } \\
\text { interações } \\
\text { hospedeiro } \\
\text { virais } \\
\text { respostas } \\
\text { imunes do } \\
\text { hospedeiro. }\end{array}$ & $\begin{array}{l}\text { Revisão de } \\
\text { Literatura. }\end{array}$ & $\begin{array}{l}\text { Pacientes com CO- VID-ige } \\
\text { diabetes exibem altas taxas } \\
\text { de citocinas e célu- las } \\
\text { inflamatórias. A enzima } \\
\text { conversora de angiotensina } 2 \\
\text { (ACE2) elevada, favorece a } \\
\text { ligação celular do SARS- } \\
\text { COV-2, dificultando a } \\
\text { resposta imunológica } \\
\text { eficiente. }\end{array}$ \\
\hline
\end{tabular}

Fonte: Autores. 
Quadro I- Artigos que abordaram os mecanismos de virulência do SARS-COV-2, bem como as complicações no prognóstico de pacientes com diabetes mellitus (continuação).

\begin{tabular}{|c|c|c|c|c|c|}
\hline $\begin{array}{l}\text { Autor/ } \\
\text { Ano }\end{array}$ & Revista & $\begin{array}{r}\text { Título do } \\
\text { Artigo }\end{array}$ & Objetivo & Métodos & Resultados \\
\hline $\begin{array}{l}\text { Torres Tamayo } \\
\text { et al., } 2020\end{array}$ & $\begin{array}{l}\text { Arch. } \\
\text { Cardiol. } \\
\text { Mex. }\end{array}$ & $\begin{array}{l}\text { Infecção por } \\
\text { Coronavírus } \\
\text { em pacientes } \\
\text { com diabetes. }\end{array}$ & $\begin{array}{l}\text { Enfocar em } \\
\text { prevalência e } \\
\text { mortalidade de } \\
\text { COVID ig na } \\
\text { população em } \\
\text { geral e com } \\
\text { diabe- } \\
\text { tes mellitus; } \\
\text { fisiopatologia de } \\
\text { SARS- para } \\
\text { CoV-2 para } \\
\text { Receptores em } \\
\text { s indivíduo } \\
\text { diabetes; a } \\
\text { resposta imune } \\
\quad \text { induzida } \\
\text { por } \quad \text { SARSCo } \\
\text { V-2 e o } \\
\text { tratamento } \\
\text { ambulatorial e } \\
\quad \text { hospitala } \\
\text { r recomendado em } \\
\text { pacientes com } \\
\text { diabetes e } \\
\text { COVID. }\end{array}$ & $\begin{array}{l}\text { Revisão de } \\
\text { Literatura. }\end{array}$ & 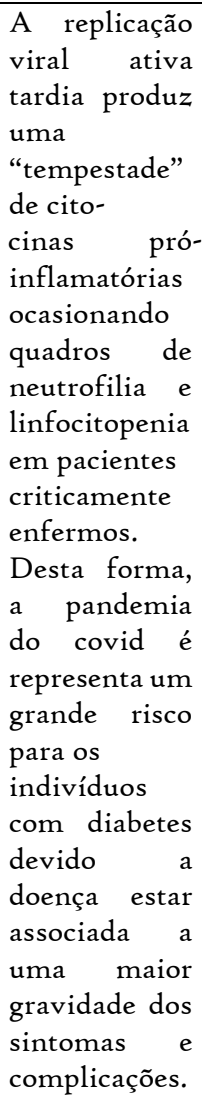 \\
\hline
\end{tabular}

Fonte: Autores. 
Quadro I- Artigos que abordaram os mecanismos de virulência do SARS-COV-2, bem como as complicações no prognóstico de pacientes com diabetes mellitus (continuação).

\begin{tabular}{|c|c|c|c|c|c|}
\hline Autor/ Ano & Revista & $\begin{array}{l}\text { Título do } \\
\text { Artigo }\end{array}$ & Objetivo & Métodos & Resultados \\
\hline $\begin{array}{l}\text { Campiol et } \\
\text { al., 202I. }\end{array}$ & $\begin{array}{l}\text { Revista } \\
\text { Multidisciplinar } \\
\text { em Saúde. }\end{array}$ & $\begin{array}{l}\text { As } \\
\text { Repercussões } \\
\text { Imunoló gicas } \\
\text { causadas } \\
\quad \text { pela } \\
\text { Infecção por } \\
\text { Coronavírus } \\
\text { ao Portador } \\
\text { de Diabetes } \\
\quad \text { Mel- } \\
\text { litus. }\end{array}$ & $\begin{array}{l}\text { Discutir } \\
\quad \text { as } \\
\text { repercussões que } \\
\text { a infec- ção por } \\
\text { CO-VID-ı9 } \\
\text { pode ocasionar } \\
\text { no sistema imu- } \\
\text { nológico do } \\
\text { indivíduo } \\
\text { portador } \\
\quad \text { de } \\
\text { Diabetes } \\
\text { Mellitus. }\end{array}$ & $\begin{array}{l}\text { Revisão de } \\
\text { Lite } \\
\text { - ratura. }\end{array}$ & $\begin{array}{l}\text { A DM quando } \\
\text { aliada ao coronavírus } \\
\text { compõese um fator } \\
\text { de risco bastante } \\
\text { elevado. A junção de } \\
\text { tais patolo-gias } \\
\text { acabam por gerar } \\
\text { graves pro- blemas, } \\
\text { compro- vando que } \\
\text { tais indivíduos ten- } \\
\text { dem a cursar com } \\
\text { deficiências } \\
\text { imunológicas, e- } \\
\text { levação de infla- } \\
\text { mação e distúr- bios } \\
\text { coagulati- vos em } \\
\text { relação aos pacientes } \\
\text { com COVID-rg não } \\
\text { diabéticos. }\end{array}$ \\
\hline $\begin{array}{l}\text { Marinho et } \\
\text { al., 2021. }\end{array}$ & $\begin{array}{l}\text { Pesquisa } \\
\text { Sociedade } \\
\text { e Desen- } \\
\text { volvimen-to. }\end{array}$ & $\begin{array}{l}\text { Interrelaçã o } \\
\text { entre Co- } \\
\text { vid-ı9 } \\
\text { diabetes } \\
\text { mellitus: } \\
\text { uma revisão } \\
\text { sistematica. }\end{array}$ & $\begin{array}{l}\text { Discutir sobre a } \\
\text { influência do } \\
\text { diabetes sobre o } \\
\text { prognóstico de } \\
\text { pacientes com } \\
\text { COVID- } \\
\text { I9. }\end{array}$ & $\begin{array}{l}\text { Revisão } \\
\text { Sistemá- } \\
\text { tica. }\end{array}$ & $\begin{array}{l}\text { Ressalta-se que, a } \\
\text { combinação de } \\
\text { fatores como a } \\
\text { obesidade, } \\
\text { hipertensão, } \\
\text { condições crônicas, } \\
\text { bem como a } \\
\text { desregulação } \\
\text { imunológica } \\
\text { disfunção alveolar e } \\
\text { endotelial e expressão } \\
\text { alterada do receptor } \\
\text { ACE2 nestes } \\
\text { pacientes contribuem } \\
\text { para a gravidade do } \\
\text { caso nestes pacientes. }\end{array}$ \\
\hline
\end{tabular}

\section{Fonte: Autores.}

Segundo Anghebem e Rego et al., (2020) os pacientes portadores de Diabetes mellitus que apresentam o vírus do SARS-CoV evoluem para quadros graves, além de possuírem uma maior taxa de morbimortalidade. A expressiva afinidade da ACE2 nas células do pâncreas constitui um elemento plausível para a complicação do 
quadro devido a este acabar afetando o metabolismo da glicose, o que favorece a hiperglicemia. Portanto, a elevação da glicemia, característica do DM, em comunhão com dentre outras tantas alterações metabólicas acabam por propiciar alterações a nível imunológico, além de favorecer um ambiente inflamatório que resultam em infecções severas e de difícil tratamento (BODE et al., 2020).

Corroborando com os dados acima mencionados, Torres-Tamayo et al., (2020) demonstraram que, de fato, a pandemia do Covid está intimamente ligada a uma maior gravidade dos sintomas e complicações para o prognóstico de pacientes com DM. Apesar de no início da pandemia os dados sobre a COVID-i9 serem escassos e inconclusivos atualmente, já se pode evidenciar que a replicação viral tardiamente acaba desencadeando uma série de citocinas próinflamatórias que acaba corroborando em quadros de neutrofilia e linfocitopenia em indivíduos em estados críticos. Portanto, pode- se verificar que, os indivíduos com diabetes é visto como um importante fator de risco para a mortalidade em pacientes infectados com coronavírus sendo semelhantes à gravidade como em indivíduos acometidos pela gripe $\mathrm{HIN}_{\mathrm{I}}$ (SCHOEN et al., 2019; SONG et al., 2019; GUPTA et al., 2020).

O processo de glicação da ACE-2 elevada pela hiperglicemia crônica, ou até mesmo por ação direta do vírus pode provocar modificações na atividade desta enzima, e isto pode ser um dos fatores para o gatilho final do estado de hiperinflamação e hipercoagulabilidade em pacientes com DM e COVID-ı. Além do mais, uma maior expressão de $\mathrm{ACE}_{2}$ no pâncreas ou até mesmo em outros órgãos (pulmão, fígado e coração) reflete ser um caminho para uma possibilidade de falência de múltiplos órgãos ocasionada como a última fase do dano viral (ANGHEBEM; REGO; PICHETH, 2020; CERIELLO, 2020; PERIC; STULNIG, 2020; TADIC; CUSPIDI; SALA, 2020).

Salienta-se que, o SARS-CoV-2 infecta as células utilizando o mesmo receptor, o ACE-2, e a partir daí atinge a parte endócrina do pâncreas provocando hiperglicemia por meio da superexpressão desta proteína pelas células das ilhotas do pâncreas, sendo estas responsáveis por hormônios como a insulina, ao qual favorece o controle glicêmico (YANG et al., 2010; SINGH et al., 2020). 
Neste sentido, ressalta-se que, a questão hiperglicêmica constitui um fator relevante neste processo, uma vez que esta é a responsável pela ativação desorganizada do sistema imunológico gerando uma imunidade mediada por células inatas desordenada com certo comprometimento dos aspectos fagocíticos pelos neutrófilos, monócitos e macrófagos, além da alteração da quimiotaxia dos neutrófilos, bem como sua atividade bactericida, liberação exagerada de citocinas pró-inflamatórias, como a interleucina- 6 (IL-6) e o fator de necrose tumoral alfaTNFa. Tais alterações imunológicas promovem infecções severas em indivíduos com DM (HIRANO; MURAKAMI, 2020; BODE et al., 2020; MA; HOLT, 2020).

Ademais, para Brito et al., (2020) a hipercitocinemia, considerada como a “tempestade de citocinas", deflagra no indivíduo uma resposta hiperinflamatória responsável pela disfunção orgânica. Deste modo, independente da via de ativação há a produção de citocinas pró-inflamatórias, além de mediadores químicos, cujo intuito é promover uma resposta antiviral efetiva. Sendo assim, diversas células, como os monócitos e macrófagos, linfócitos e neutrófilos acabam por migrar para o epitélio pulmonar para tentar conter o SARS-CoV-2. Entretanto, se tais tentativas de limitar a infecção ocorrem de maneira elevada, as consequências sera os efeitos oxidativos e inflamatórios que de maneira inespecífica acabam gerando danos secundários aos tecidos funcionais e são infectados.

Muniyappa e Gubbi (2020), relataram que pacientes com SARS-CoV-2 exibem contagens de células $\mathrm{T} \mathrm{CD}_{4}$ e CD8 diminuídos, além de níveis de citocinas elevadas, corroborando com os dados dos autores acima. Inúmeros pacientes que possuem casos severos da COVID-ı9 demonstraram concentrações séricas expressivas de citocinas pró- inflamatórias como a IL-6

(interleucina-6) e IL-i $\beta$, bem como IL-8, GM-CSF, MCPı, TNF e dentre outras. A ação dessas variadas citocinas estimula um ambiente favorável para o desencadeamento do processo inflamatório (WU et al., 2020; YANG et al., 2020).

Esses mais variados complexos proteicos estão intimamente ligados na regulação da resposta imune e envolvidos na indução da patologia por SARSCoV e MERS-CoV, principalmente as proteínas celulares $\mathrm{MYCBP}_{2}, \mathrm{NCOA} 6, \mathrm{PIK}_{3} \mathrm{C}_{2} \mathrm{G}$ e KLFio, aos quais interagem com o domínio do terminal carboxila da proteína za e 
estão envolvidas no controle da inflamação, uma das causas de virulência do SARSCoV. Sendo assim, estas acabam por modular a virulência viral (RODRIGUES, 2018).

Segundo Marinho et al., (202I) diversos mecanismos têm sido verificados na correlação do mau prognóstico em pacientes diabéticos, o que favorecem para severidade da doença. A desregulaçao imunológica causada pelo SARS- CoV-2 em pacientes diabéticos pode desencadear a produção de citocinas e quimiocinas que podem resultar em uma inflamação que acabam por contribuir em destruição das células pancreáticas, gerando estados hiperglicêmicos, reduzindo assim, o mecanismo de rastrear a infecção e contê-la, dificultando o processo de cura e prolongando a recuperação, além de induzir a replicação viral (GUO et al., 2020; MALLAPATY, 2020).

A junção de ambas as patologias, COVID e diabetes, constituem um grande fator de risco. Isto se dá em virtude das deficiências imunológicas causadas pela elevada produção de interleucina-6, proteína $C$ reativa e dímero $D$ (índice de coagulação, além da linfopenia e neutrofilia, o que acaba prejudicando no sistema imunológico, tornando-se amplificada a atuação viral e desregulando todo o metabolismo de glicose, uma vez que ocorre grandes alterações nas ilhotas do pâncreas. Portanto, a DM é umas das comorbidades que mais favorece a morbimortalidade e desfechos severos na COVID-19, além de promover a deficiência imunológica elevada tornando o doente suscetível as mais variadas infecções (CAMPIOL et al., 202I).

Em virtude do exposto, os indivíduos com diabetes e infectados com SARSCoV-2, além das consequências oriundas da perturbação do estado inflamatório crônico, este acaba estimulando a produção de glicose dos hepatócitos, além de reduzir a atividade da insulina conferindo resistência insulínica bem como ao aparecimento de quadros de cetoacidose. Adicionado-se a isso, as manifestações extrapulmonares da COVID-I9 também podem estar atreladas às complicações diabéticas, como é o caso da diminuição da função dos rins, estado pró- trombótico e de coagulação elevados, problemas cardíacos e injúria nos hepatócitos. Já o fator 
obesidade, provoca uma grande liberação de citocinas, fatores de necrose tumoral e interleucinas, o que favorece uma resposta inflamatória (GUPTA et al., 2020).

A patogenicidade do SARS-CoV-2 confere aos pacientes portadores de DM um maior índice de mortalidade e necessidade de cuidados mais intensos, devido a estes serem mais susceptíveis a infecções em virtude do processo inflamatório e estresse oxidativo que acaba afetando as taxas glicêmicas prejudicando toda a homeostase corporal, bem como a sensibilidade periférica à ação insulínica, uma vez que a adaptação metabólica nos pacientes diabéticos demonstra estar prejudicada no contexto do COVID-ı, o que predispõe o indivíduo à infecção, surgimentos de complicações, além de estar atrelado a um pior prognóstico e controle de glicemia (ANGHEBEM; REGO; PICHETH, 2020; AQUINO, 2020; JAMES et al., 2020).

\section{CONCLUSÃO}

Diante disso, foi demonstrado que a inflamação metabólica provocado pelo SARS-CoV-2 gera grandes repercussões negativas no prognóstico de paciente portador de DM. Ressalta-se que, os pacientes diabéticos infectados apresentam além do comprometimento da imunidade advindo do estado inflamatório crônico, estes acabam estimulando hormônios que promovem não somente a produção de glicose hepática e a liberação de insulina reduzida, mas também a cetoacidose e resistência à insulina. Portanto, tais manifestações acabam por provocar a redução da habilidade do corpo em rastrear a infecção e combatê-la, dificultando assim, o processo de cura e recuperação do indivíduo.

\section{REFERÊNCIAS}

ANDERSEN, K. G. et al. A origem proximal do SARS-CoV-2. Nat Med, v. 26, n. 4, p. $450-452,2020$.

ANGHEBEM, M. I.; REGO, F. G. M.; PICHETH, G. COVID-i9 e Diabetes: a relação entre duas pandemias distintas. RBAC, v.52, n.2, p.9-154, 2020.

AQUINO, P. A. G. Q.; SILVA, A. F.; OLIVEIRA, A. V. A.; ANDRADE, M. B.; NUNES, M. H.; SOARES, V. O. V. Influência do DMz e do controle glicêmico no prognóstico de pacientes infectados por COVID-I9. Brazilian Journal of Health Review, v.3, n.4, p.III20-III30, 2020. 
BARBOSA, S. A.; CAMBOIM, F. E. F. Diabetes mellitus: cuidados de enfermagem para controle e prevenção de complicações. João Pessoa (PB)[Internet], v. I6, n. 3, p. 404417, 2016.

BODE, B. et al. Glycemic characteristics and clinical outcomes of COVID-i9 patients hospitalized in the United States. Journal of diabetes science and technology, v. I4, n. 4, p. 813-82I, 2020.

BRASIL. Ministério da Saúde. Protocolo de manejo clínico para o novocoronavírus (2019-nCoV) (2020). Disponível em:<

https://portalarquivos2.saude.gov.br/images/pdf/2020/fevereiro/II/protocolomanej o- coronavirus.pdf $>$. Acesso em: io de Março de 2020 .

BRASIL. Ministério da Saúde. Coronavírus: o que você precisa saber e como prevenir o contágio (2020). Disponível em:〈https://saude.gov.br/saude-de-az/coronavírus〉.

Acesso em: io de Março de 202I.

BRITO, S. B. P.; BRAGA, I. O.; MORAES, M. M.; CUNHA, C. C.; LEÃO, S. C.;

TAKENAMI, I. Mecanismos imunopatológicos envolvidos na infecção por

SARSCoV-2. J. Bras. Patol.Med. Lab., v.56, p.I-Io, 2020.

CAMPIOL, N. L. et al. As Repercussões Imunológicas causadas pela Infecção por

Coronavírus ao Portador de Diabetes Mellitus.Revista Multidisciplinar em Saúde, v.2,

n.2, p.33-33, 2021.

CERIELLO, A. Management of diabetes today: An exciting confusion. Diabetes research and clinical practice, v. 162, p. 108129-108129, 2020.

FREITAS, A. H. Reflexões sobre a Pesquisa Acadêmica: Revisão Bibliográfica, Vivência e conhecimento. Palíndromo, v. 8, n. 15, p. 074-082, 2016.

GUAN, W. J.; NI, Z. Y.; HU, Y.; LIANG, W. H.; OU, C. Q.; HE, J. X. et al; China Medical Treatment Expert Group for Covid-r9. Clinical characteristics of coronavirus disease 2019 in China.N Engl J Med., v.382, n.18, p.1708-1720, 2020.

GUO, W.; LI, M.; DONG, Y.; ZHOU, H.; HU. Diabetes is a risk factor for the progression and prognosis of COVID-19. Diabetes/metabolism research and reviews, v. 36, n. 7, p.3319, 2020.

GUPTA, R. et al. Clinical considerations for patients with diabetes in times of COVID-r9 epidemic.Diabetes \& metabolic syndrome, v. I4, n. 3, p. 211, 2020.

GUPTA, A. et al. Extrapulmonary manifestations of COVID-I9.Nature medicine, v. 26, n. 7, p. 1017-1032, 2020. 
HIRANO, T.; MURAKAMI, M. COVID-ig: a new virus, but a familiar receptor and cytokine release syndrome. Immunity, v. 52, n. 5, p. 731-733, 2020.

HUANG, C. et al. Clinical features of patients infected with 2019 novel coronavirus in Wuhan, China. The lancet, v. 395, n. 10223, p. 497-506, 2020.

JAMES, M. et al. Pharmacologic treatments for coronavirus disease 2019 (COVIDiq).JAMA, v.323, n.18, p.1824-I836, 2020.

MALLAPATY, S. Evidence suggests the coronavirus might trigger diabetes. Nature, v.583, p. I6-17, 2020.

MA, R. C. W.; HOLT, R. I. G. COVID-I9 and diabetes.Diabet Med., v.37, n.5, p.723$725,2020$.

MARINHO, F. P.; LOYOLA, I. S.; MONTEIRO, I. O. F.; CASTRO, T. M.; CARVAlHO, M. G. S.; GARCIA, J. A. D.; SILVÉRIO, A. C. P.; SANTOS, G. B. Inter-relação entre COVID-ıg e diabetes mellitus: uma revisão sistemática. Research Society and Development, v.Io, n.2, p.I-I4, 202I.

MUNIYAPPA, R.; GUBBI, S. COVID-i9 pandemic, coronaviruses, and diabetes mellitus.American Journal of Physiology-Endocrinology and Metabolism, v. 318, n. 5, p. $\mathrm{E}_{736}-\mathrm{E}_{741}$, 2020.

NEVES, M. O. A Importância da Investigação Qualitativa no processo de formação continuada de Professores: Subsídios ao exercício da Docência. Revista Fundamentos, v.2, n.I, p.17-31, 2015.

NOBRE, A. F. S. et al. Primeira detecção de coronavírus humano associado à infecção respiratória aguda na Região Norte do Brasil. Rev. Panz, v.5, n.2, p.374I, 2014.

PERIC, S.; STULNIG, T. M. Diabetes and COVID-I9.Wiener Klinische Wochenschrift, v. 132, n. 13, p. 356-361, 2020.

RODRIGUEZ, C. C. Papel de lasviroporinas E, za y 8a delcoronavirusdel

SARS en replicación y virulência (2018). Tese de Doutorado. Universidad Autónoma de Madrid. Disponível em: $<$ https://repositorio.uam.es/handle/10486/686207?show =full $>$. Acesso em: 15 de Junho de 2020.

SCHOEN, K. et al. Spectrum of clinical and radiographic findings in patients with diagnosis of $\mathrm{HIN}_{\mathrm{I}}$ and correlation with clinical severity. BMC infectious diseases, v. I9, n. I, p. I-8, 2019. 
SINGH, A. K. et al. Diabetes in COVID-I9: Prevalence, pathophysiology, prognosis and practical considerations. Diabetes \& Metabolic Syndrome: Clinical Research \& Reviews, v. I4, n. 4, p. 303-310, 2020.

SOCIEDADE BRASILEIRA DE DIABETES. Diretrizes da Sociedade Brasileira de Diabetes 2019-2020. Diretrizes da Sociedade Brasileira de Diabetes 2019-2020 (2019). Disponível em:< https://www.diabetes.org.br/profissionais/images/DIRETRIZES-COMPLETA2or92020.pdf $>$. Acesso em: io de Março de 2021.

SONG, Z. et al.From SARS to MERS, thrusting coronaviruses into the spotlight.viruses, v. II, n. I, p. 59, 2019.

TADIC, M.; CUSPIDI, C.; SALA, C. COVID_is and diabetes: Is there enough evidence?.The Journal of Clinical Hypertension, v. 22, n. 6, p. 943-948, 2020.

TORRES-TAMAYO, M. et al. Coronavirus infection in patients with diabetes.Archivos de cardiologia de Mexico, v. 90, p. 67-76, 2020.

ZHU, N. et al. A novel coronavirus from patients with pneumonia in China, 2019.New England journal of medicine, v.382-, n.8, p.727-733, 2020.

WU, C. et al. Risk factors associated with acute respiratory distress syndrome and death in patients with coronavirus disease 2019 pneumonia in Wuhan, China. JAMA internal medicine, v. I8o, n. 7, p. 934-943, 2020.

YANG, J. et al. Binding of SARS coronavirus to its receptor damages islets and causes acute diabetes. Acta diabetologica, v. 47, n. 3, p. 193-199, 2010.

YANG, J. K.; FENG, Y.; YUAN, M. Y.; YUAN, S. Y.; FU, H. J.; WU, B. Y. et al. Plasma glucose levels and diabetes are independent predictors for mortality and morbidity in patients with SARS. Diabet Med., v.23, n.6, p.8-623, 2006.

YANG, X. et al. Clinical course and outcomes of critically ill patients with SARSCoV2 pneumonia in Wuhan, China: a single-centered, retrospective, observational study. The Lancet Respiratory Medicine, v. 8, n. 5, p. 475-48I, 2020. 